\title{
Promoting Autonomy in a Smart Home Environment with a Smarter Interface
}

\author{
Brennan, CP., McCullagh, PJ., Galway, L., \& Lightbody, G. (2015). Promoting Autonomy in a Smart Home \\ Environment with a Smarter Interface. In Unknown Host Publication (pp. 5032 -5035). IEEE. \\ https://doi.org/10.1109/EMBC.2015.7319522
}

Link to publication record in Ulster University Research Portal

\author{
Published in: \\ Unknown Host Publication
}

Publication Status:

Published (in print/issue): 25/08/2015

DOI:

10.1109/EMBC.2015.7319522

\section{Document Version}

Publisher's PDF, also known as Version of record

\section{General rights}

Copyright for the publications made accessible via Ulster University's Research Portal is retained by the author(s) and / or other copyright owners and it is a condition of accessing these publications that users recognise and abide by the legal requirements associated with these rights.

\section{Take down policy}

The Research Portal is Ulster University's institutional repository that provides access to Ulster's research outputs. Every effort has been made to ensure that content in the Research Portal does not infringe any person's rights, or applicable UK laws. If you discover content in the Research Portal that you believe breaches copyright or violates any law, please contact pure-support@ulster.ac.uk. 


\title{
Promoting Autonomy in a Smart Home Environment with a Smarter Interface*
}

\author{
C. P. Brennan, P. J. McCullagh, L. Galway and G. Lightbody ${ }^{1}$
}

\begin{abstract}
In the not too distant future, the median population age will tend towards 65; an age at which the need for dependency increases. Most older people want to remain autonomous and self-sufficient for as long as possible. As environments become smarter home automation solutions can be provided to support this aspiration. The technology discussed within this paper focuses on providing a home automation system that can be controlled by most users regardless of mobility restrictions, and hence it may be applicable to older people. It comprises a hybrid Brain-Computer Interface, home automation user interface and actuators. In the first instance, our system is controlled with conventional computer input, which is then replaced with eye tracking and finally a BCI and eye tracking collaboration. The systems have been assessed in terms of information throughput; benefits and limitations are evaluated.
\end{abstract}

\section{INTRODUCTION}

During the twentieth century the life expectancy of the worlds most affluent populations rose dramatically, leading to an increase in the mean age of the population worldwide [1]. According to the UK parliament, 10 million of its current residents are over the age of 65 , with projections anticipating this figure to rise by a further 5.5 million within the next twenty years. Indeed, it is expected that by 2050, 19 million of the UK population will be over this age [2]. Such an increase in longevity has become a key societal challenge but how can we, as a society, improve the quality of life and general welfare of this demographic? The advancements in modern medicine, healthcare, hygiene, food supply, nutrition, and technology are all contributing factors toward this increase in life expectancy. However, serious societal and economic concerns arise when the largest proportion of the population is over 65 ; the age at which people become less productive from a work perspective and in addition the need for healthcare dramatically increases. Consequently, the increased burden placed upon caregivers must be addressed, especially as the number of caregivers will be greatly outnumbered by the number of those that need care [3]. Subsequently, one proposed solution is to provide technological innovation in order to optimize self-sufficiency. While it is common for old age to be seen as a time of increased dependency, home automation has the potential

\footnotetext{
*This work was supported by the Department of Education and Learning and the University of Ulster

${ }^{1}$ C. P. Brennan, P. J. McCullagh, L. Galway, and G. Lightbody are with the Computer Science Research Institute, Faculty of Computing and Engineering, University of Ulster, Jordanstown Campus, Shore Road, Newtownabbey, Co. Antrim, BT37 0QB brennan-c15@email.ulster.ac.uk; ppj.mccullagh; l.galway; g.lightbody\}@ulster.ac.uk
}

to increase autonomy and empower the users capability to interact with day-to-day activities in their home environment.

Over the last 30 years, solutions such as stair lifts and automatic doors have become prominent within homes, offices and public places. The next phase is the integration and application of Information and Communication Technology (ICT) advancements within the home, thereby facilitating the deployment and creation of smart environments [4]. Within this domain a users capabilities may be augmented to facilitate interaction with smart devices and applications. A user could, for example, interact with a visual interface to turn off an upstairs light or set a timer to automatically lock their home in the evening. Such innovations may improve self-sufficiency, independence and wellbeing. As with all implementations in a domestic smart environment, there exists a challenge in terms of how to facilitate interaction [5]. Controlling an application on a visual interface is one such approach, which can be easily adapted to meet the requirements of specific users.

For most users, a conventional input modality such as a mouse and keyboard suffice but for users that have difficulties and restrictions with the operation and use of technology and those that have reduced mobility, assistive technology is a potential solution. Alternative enabling technologies are (i) the Brain Computer Interface (BCI) and (ii) the eye tracker. With a BCI the system must utilize activity directly recorded from the brain that can be intentionally modulated and processed in real time to produce a communication or control signal that is validated by feedback [6]. A user could interact simply by looking at a screen using an eye tracking component, or they could utilize Brain-Neuronal Computer Interface (BNCI) to complete a task. A BNCI is similar to a Brain Computer Interface (BCI), but includes devices that monitor other physiological signals as well, such as Electrooculography (EoG), Electromyography (EMG) or heart rate [7].

In this paper, potential technologies and input modalities for home automation are discussed and contrasted. We present an architecture and visual interface to facilitate smart home interaction and conclude by evaluating the implemented system in terms of information throughput.

\section{Home Automation Technologies}

The ability to provide automatic and remote control of basic home functions and features, such as air conditioning, cookers, microwave ovens, heating, lighting, media devices, opening/closing windows and doors, and security systems 
is known as home automation [8]. The fundamental components of such a system include a computer and software, cables and wireless links for network connectivity, sensors, and the devices and applications to be controlled [9]. Domotic control is the capability to communicate with devices in a smart environment. Protocols include Control4, Pi-mote, $\mathrm{X} 10$, and Z-Wave.

Control4 is marketed as a full home automation system, software is proprietary and cannot be customized by a third party. It does not support a range of input modalities and control is limited to a smart phone application or Internet browser, neither of which could support eye tracking or the use of BNCI devices.

$\mathrm{Pi}$-mote is one alternative method of providing domotic control at a fraction of the cost. Its Open Source code facilitates use of a wide range of input modalities. In the implementation discussed herein, a Raspberry Pi is fitted with a Radio Frequency (RF) controller board, which transmits signals to remote sockets around the home. As expected there is a trade-off between performance and cost; the Pi-mote can only transmit 10 different codes. This approach limits the number of devices that can be controlled by each Raspberry $\mathrm{Pi}$, although more than one socket may be controlled by the same transmission code, permitting concurrent switching of lights for example.

An alternative method is the industry standard communication protocol, X10. By using power line carrier control, X10 can send digital data that consists of an address and a command, through household electrical wiring circuits. However, one of the limitations of this technology is that other electrical devices can create electrical interference on the power lines, thereby preventing X10 signals from being received correctly by its modules. Similar to Pi-mote, X10 does not facilitate more complex forms of communication, such as changing the television channel [10].

Z-Wave permits devices within a smart environment to be controlled using a smart phone, tablet or PC. It is a wireless technology that makes standard home fixtures and fittings, such as door locks, lights, and thermostats "smart". Z-Wave facilitates the ability to interact with household devices through an online gateway, which provides encrypted communication via AES128 and external interaction through VPN to provide services such as lighting, locks, remote control, security, sensors, smart meters, thermostats, USB, and motor control. Custom input modalities may be utilized in order to send packets to the gateway that subsequently execute relevant commands. However, one of the main limitations of the Z-Wave is its cost, nearly three times the price of X10 devices. Furthermore, in order to program bespoke software, an expensive developers kit is necessary.

An aspect for the effective and successful use of domotic control is user interaction. A mouse, and keyboard, eye tracker, and BNCI device are all viable methods of input, which can accommodate the wide ranging abilities of users. For the most part, a mouse and keyboard may suffice as an appropriate interaction mechanism. However, systems within a smart environment are often controlled through a television set in the living room, which makes connecting via a wired mouse and keyboard problematic. A more natural and intuitive system may incorporate eye tracking as an alternate input modality, since a user could control an application simply by looking at it. However, this approach is hindered by the limitations associated with eye tracking technology.

\section{Systems ARChitecture}

The implemented system can receive input from either a mouse or keyboard, the EyeTribe tracker, or the Emotiv EPOC and EyeTribe Tracker combined. The data acquired from each input modality is processed through software applications and SDKs and transmitted to the visual menu application, which interacts with applications and devices in the smart environment. Figure 1 provides an overview of the system architecture.

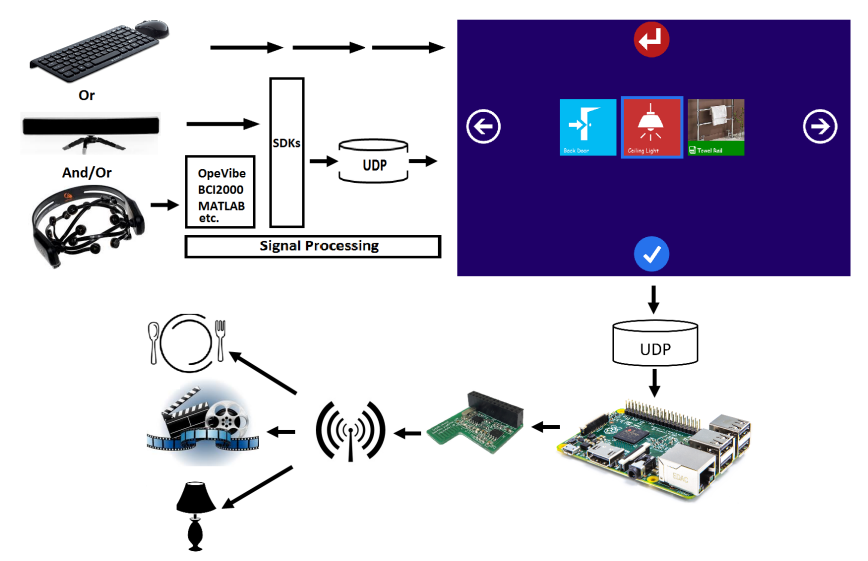

Fig. 1. The systems architecture. Input signals are generated using a mouse and keyboard, an eye tracker, or an EEG headset. The signals are then processed through specialist software and SDKs. The data is then encapsulated in UDP packets and transmitted to the visual menu application. Upon selection the visual menu application transmits another UDP packet to a Raspberry Pi, which transmits a radio frequency to devices \& applications

The mouse and keyboard approach acquires input based on cursor control, mouse click, or key press and passes this information directly to the visual menu application. Whereas input from the EyeTribe tracker approach is more complex. It makes use of a camera and infrared light to measure eye activity and extrapolate on-screen gaze-based coordinates, which are output as numerical values in the SDK. These values are processed into relevant commands, encapsulated in UDP packets and transmitted to the visual menu application. In the case of the Emotiv EPOC, an input signal is acquired by measuring voltage fluctuations along the scalp across 14 channels, a process known as Electroencephalogram (EEG). These fluctuations are output as numerical values in their respective SDK where different values from alternative channels are representative of different components, such as teeth grind, smile, eye activity and current action power levels etc. If the value for a teeth grind component is within a predefined threshold and the gaze-based coordinates are in a defined location on the screen then a string is encapsulated within a 
UDP packet and transmitted to the visual menu application to execute a command.

Regardless of which method of input is utilized, the menu system can be navigated to select other applications and events within the smart environment. When a device is selected another UDP packet is sent across the network to the Raspberry $\mathrm{Pi}$, which is pre-configured to listen for UDP packets that contain specific codes. The Raspberry Pi is fitted with the Pi-mote controller board and programmed using the Python programming language. Once a specific code is received, the Raspberry $\mathrm{Pi}$ transmits a radio frequency to the target devices, thus controlling, lights, air conditioning, and any other devices in the smart home that employ the Pimote sockets. In addition to facilitating control of appliances, this system also provides the facility for interaction with multimedia and communication through iconography.

\section{METHODS}

In order to assess the system in terms of performance, the Information Transfer Rate (ITR) for each of the three input modalities: 1) Mouse and Keyboard (N=1); 2) EyeTribe Tracker $(\mathrm{N}=16)$; and 3) Emotive EPOC and Eyetribe Tracker combined $(\mathrm{N}=12)$ was calculated using the following equation [11]:

$$
I T R=\left(\log _{2} M+P \log _{2} P+(1-P) \log _{2}[(1-P) /(M-1)]\right)
$$$$
*(60 / T)
$$

where $\mathrm{M}$ is the number of choices, $\mathrm{P}$ is the accuracy of target detection and $\mathrm{T}$ (seconds/selection) is the average time for a selection. Approval to carry out experimental procedures on human participants was granted by the Ethics Committee of the Faculty of Computing and Engineering in Ulster University.

All experiments were conducted on a control group ranging from 23-57 years of age. Participants included students, lecturers and the general public as long as they were not under 18 years old or part of a vulnerable group. In the experimentation phase, each user had to complete three tasks: Task one (domotic control): navigate the on-screen menu system to turn on the dining room light; Task two (multimedia control): navigate the menu system to play a video and then stop playback; and Task three (communication by iconography): navigate the menu system and select the appropriate icon to indicate hunger. Each task was timed and all selections were recorded. Figure 2 shows a representative subject using the system within a smart environment. The user is interacting with an application on the living room TV by using the EyeTribe Tracker and the Emotiv EPOC as a collaborative assistive technology.

\section{RESUlTs}

As mentioned previously, a range of different input modalities can be employed to control the visual menu system. Using the mouse and keyboard as an input modality worked as expected and by simply controlling a cursor, users were able to successfully make a selection by pointing and clicking. For a representative subject, the mean Accuracy and

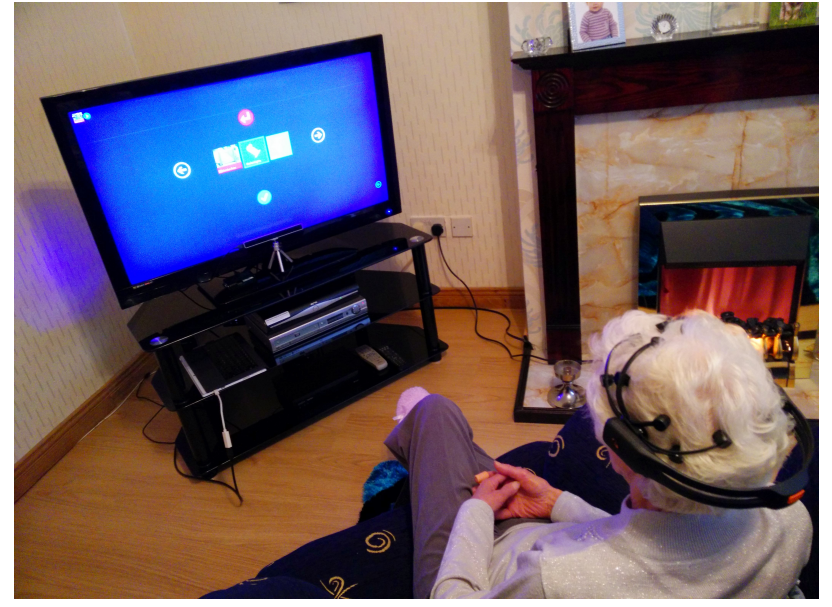

Fig. 2. A representative subject using the implemented system in order to complete the three tasks within a smart home using the Emotiv EPOC and the EyeTribe tracker together

ITR for all three tasks was $100 \%$ and $317.60 \mathrm{bits} / \mathrm{min}$ respectively. This was the highest Accuracy and ITR obtained from all modalities tested. Nevertheless, some users do not have a choice in which modalities they can and cannot use. For this reason, an eye tracking-based approach was also tested on a control group of 16 users, obtaining a mean Accuracy and ITR of $91.70 \% \pm 4.45 \%$ and $178.31 \pm 20.50$ bits/min, respectively. The same experiment was then carried out using collaborative input modalities consisting of eye tracking and EEG. The Emotiv EPOC was used to measure EEG whereby a BNCI component was extracted and used as a condition in the program. This was the essential criteria that instructed the system to begin looking for gaze-based coordinates in predefined quadrants of the screen. In this case a mean Accuracy of $95.45 \% \pm 4.54 \%$ and mean ITR of and $210.10 \pm 21.13 \mathrm{bits} / \mathrm{min}$ was achieved for 12 users. Each user reported full control of the system, as they were able to pause, read, talk and think in between each task without controlling the system unintentionally.

\section{Discussion}

All tested input mechanisms provide fast and fluent control of the application and subsequent events within the smart environment. However, a comparison can be made with regard to the advantages and disadvantages of each approach. The input modality with the highest performance, in terms of ITR, the mouse and keyboard, suffers from issues of accessibility. Not all users will be able to utilize this method for input and, as such, an alternative is to employ eye tracking. This is an intuitive input mechanism, since users can perform selections simply by looking at a target icon on the user interface.

\section{A. Eye Tracking}

The problem that arises, in this case, is one of intentional selection. The implemented system utilizes dwell-time in order to perform selections. By focusing their gaze on an 
icon, the user can select it but this creates a serious concern, especially where domotic control is exercised. When intentionally controlling the interface, users can achieve an accuracy of nearly $100 \%$. Once a user stops controlling the interface, however, the eye tracker will continue to transmit gaze-based coordinates to the application, which will subsequently activate events within the smart environment unintentionally. This is fine in experimental conditions but not in a real world scenario, as unintentional selections will trigger intolerable events in the local environment. Lights would be flashing on and off, doors and windows would be opening and closing, and security systems would be activating and deactivating, which is certainly not ideal. Alternatively, when a user finishes with intentional domotic control they are likely to change the television channel, which is also facilitated by the application. Doing so does not stop the eye tracker, as the user may still need to interact with the smart environment at a later time. So, while the user is watching the television events will be unintentionally triggered in the smart environment, yet again. Eye tracking suffers from intended selection restrictions. In a dwell-time based system, how does the application differentiate between dwell times that are representative of a selection and those that are not (when a user pauses to read or think, for example.) Inm addition eye trackers are also hindered by user location. Users have to directly face the device in which they are interacting. If they are sitting at an angle or in a different location from when the eye tracker was calibrated, control will be limited at best.

\section{B. Hybrid Solution}

In order to prevent such situations from occurring, an Emotiv EPOC was integrated as a "switch", which utilizes a teeth clench component to select the icon a user is focusing their gaze upon. This approach mitigates the undesirable effects of the eye tracking only system but is endowed with set-up restrictions, especially for users with reduced mobility and a lack of motor control. If a user does not have the motor control necessary to use a mouse and keyboard, it is somewhat unlikely that they will have the ability to self-apply the EEG device. In extreme cases, where mobility is seriously reduced, a BNCI device may be coupled with an eye tracker to provide input. The devices that could be incorporated in a domestic smart environment include generic keyboards and mouses, Tobii X60, EyeTribe Tracker, Emotiv EPOC, Emotiv Insight, Neuroskys Mindwave and g.Tecs g.Nautilus dry electrode BCI system. The EPOC, Insight, Mindwave and g.Nautilus are all examples of wireless Electroencephalogram (EEG) acquisition devices, each with its own specifications and limitations. The EPOCs electrodes are water based and therefore require experimenter/caregiver assistance and as such may not be suitable for self-application in the home. The other three devices utilise dry electrodes to acquire biosignals recorded directly from the scalp. The g.Nautilus is a high performing device but it is not cost effective whereas the Mindwave and Insight are much more affordable but may not provide sufficient signals to offer control.

\section{CONCLUSION}

In this work we have shown the feasibility of using collaborative input modalities in order to provide home automation. Each of the devices utilized are cost-effective, highly usable, and sufficiently accurate, which makes them particularly suited to deployment outside of laboratory conditions. The exception, however, is the Emotiv EPOC, as it requires experimenter or caregiver assistance to set-up correctly. Given that the developed system is aimed at older people and those with reduced mobility, the EPOC is not a suitable choice of assistive technology when promoting active ageing. However it did outperform the eye tracking only approach in terms of both accuracy and ITR. The hybrid approach achieved an accuracy of $95.45 \%$ and an ITR of $210.10 \mathrm{bits} / \mathrm{min}$ as opposed to an accuracy of $91.70 \%$ and an ITR of $178.31 \mathrm{bits} / \mathrm{min}$ for the eye tracking only approach. The hybrid approach also excelled in terms of its ability to only provide interaction when desired by the user. The results of this suggest that such hybrid systems are feasible. In future work the EPOC could be replaced with a dry electrode headset that can offer similar functionality and be easily selfapplied or the system could be further tested on users with an acquired brain injury.

\section{REFERENCES}

[1] R. Suzman and J. Beard, "Global Health and Aging," US Department of State, pp. 1-32, 2011. [Online]. Available: papers2: //publication/uuid/150A301A-0A61-489C-80D4-3C889BE672E2

[2] T. Rutherford, "Population ageing: statistics," House of Commons library, no. February, 2012. [Online]. Available: http://www.parliament. uk/business/publications/research/briefing-papers/SN03228.pdf

[3] T. Day, "Guide to Long Term Care Planning," 2014. [Online]. Available: http://www.longtermcarelink.net/eldercare/caregiving.htm

[4] V. Kaptelinin, "Managing project contexts : Interaction history as a resource," in Cognitive Science Research, University of Sussex, 2005, pp. 1-6. [Online]. Available: http://www.pervasive.ifi.lmu.de/workshops/ w8/papers/echise2005-s08-ManagingProjectContexts-Kaptelinin.pdf

[5] D. Surie, T. Pederson, and L.-E. Janlert, "A Smart Home Experience Using Egocentric Interaction Design Principles," in 2012 IEEE 15th International Conference on Computational Science and Engineering, 2012, pp. 656-665. [Online]. Available: http: //ieeexplore.ieee.org/lpdocs/epic03/wrapper.htm?arnumber $=6417354$

[6] BrainAble, Future BNCI: A Roadmap for Future Direction in Brain / Neuronal Computer Interaction Research, 2012. [Online]. Available: http://www.brainable.org/Documents/Future $\backslash$ BNCI $\backslash$ Roadmap.pdf

[7] C. Carmichael and P. Carmichael, "BNCI systems as a potential assistive technology: ethical issues and participatory research in the BrainAble project." Disability and rehabilitation. Assistive technology, vol. 9, no. 1, pp. 41-7, Jan. 2014. [Online]. Available: http://www.ncbi.nlm.nih.gov/pubmed/24308848

[8] A. Z. Alkar and U. Buhur, "An Internet Based Wireless Home Automation System for Multifunctional Device," IEEE Transactions on Consumer Electronics, vol. 51, no. 4, pp. 1169-1174, 2005. [Online]. Available: http://ieeexplore.ieee.org/xpl/articleDetails.jsp? arnumber $=1561840$

[9] D. J. Cook and S. K. Das, "How Smart are our Environments ? An Updated Look at the State of the Art The Role of Physical Components in Smart Environ- ments," Journal of Pervasive and Mobile Computing, vol. 3, pp. 53-73, 2007.

[10] A. Brush, B. Lee, and R. Mahajan, "Home automation in the wild: challenges and opportunities," in Proceedings of the SIGCHI Conference on Human Factors in Computing Systems, 2011, pp. 21152124. [Online]. Available: http://dl.acm.org/citation.cfm?id=1979249

[11] S. Gao, Y. Wang, X. Gao, and B. Hong, "Visual and auditory braincomputer interfaces." IEEE transactions on bio-medical engineering, vol. 61, no. 5, pp. 1436-47, May 2014. [Online]. Available: http://www.ncbi.nlm.nih.gov/pubmed/24759277 